\title{
Compreensão da Leitura, Atitudes de Leitura e Desesperança em Universitários
}

\author{
Reading comprehension, reading atittudes \\ and hopelessness in university students \\ Comprensión de la lectura, actitudes de \\ lectura y desesperanza en universitarios
}

Katya Luciane de Oliveira

Universidade São Francisco

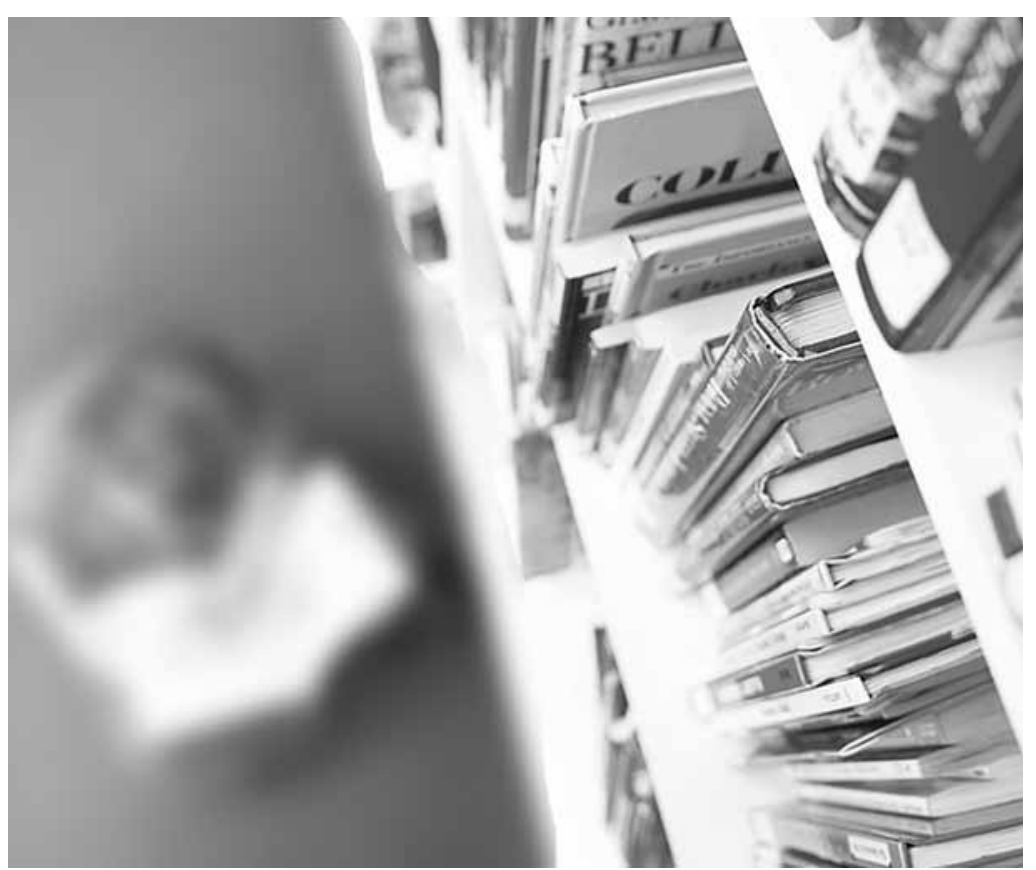


Resumo: O objetivo deste estudo foi levantar relações entre a compreensão e as atitudes em relação à leitura e a desesperança em estudantes universitários. Participaram 261 universitários dos cursos de administração, radiologia e Psicologia de universidades privadas dos Estados de São Paulo e Minas Gerais. Um texto de 250 vocábulos, preparado segundo a técnica de Cloze, em sua versão tradicional, em que são omitidos todos os quintos vocábulos do texto, uma escala de atitudes de leitura e uma escala de desesperança foram utilizadas para tal fim. A aplicação foi coletiva, e os dados, submetidos à estatística inferencial. A diferença estatisticamente significativa na pontuação dos instrumentos, considerando o gênero e a idade dos participantes, foi evidenciada. Foi também possível levantar as relações significativa e negativa entre a pontuação na Escala de Atitudes de Leitura e os escores na Escala de Desesperança. Os dados são discutidos em termos de suas implicações para a área de avaliação psicoeducacional.

Palavras-chave: Leitura. Técnica de Cloze. Desesperança. Universidade.

\begin{abstract}
The objective of this survey is to associate reading comprehension, reading attitudes and hopelessness in university students. 261 university students who attended the administration, radiology and Psychology courses in private universities in São Paulo and Minas Gerais participated in the research. A text with 250 words, prepared according to Cloze's technique, in its traditional version, a reading attitudes scale and a hopelessness scale were used. The application was collective and the data were submitted to inferential statistics and showed clearly a statistically significant difference in the punctuation of the instruments considering the participants' gender and age. It was also possible to associate significant and negative relations between the punctuation in both scales. The data were discussed in terms of their implications for the psycho educational assessment area.
\end{abstract}

Keywords: Reading. Cloze's technique. Hopelessness. University.

Resumen: El objetivo de este estudio fue levantar relaciones entre la comprensión y las actitudes con relación a la lectura y la desesperanza en estudiantes universitarios. Participaron 261 universitarios de los cursos de administración, radiología y Psicología de universidades privadas de los Estados de Sao Paulo y Minas Gerais. Un texto de 250 vocablos, preparado según la técnica de Cloze, en su versión tradicional, en que son omitidos todos los quintos vocablos del texto, una escala de actitudes de lectura y una escala de desesperanza fueron utilizadas para tal fin. La aplicación fue colectiva, y los datos, sometidos a la estadística inferencial. La diferencia estadísticamente significativa en la puntuación de los instrumentos, considerando el género y la edad de los participantes, fue evidenciada. Fue también posible levantar las relaciones significativa y negativa entre la puntuación en la Escala de Actitudes de Lectura y los resustados en la Escala de Desesperanza. Los datos son discutidos en términos de sus implicaciones para la área de evaluación psicoeducacional.

Ler criticamente requer uma conexão mental entre o sistema cognitivo e as partes que compõem o texto, pois a essência da leitura é a sua compreensão, uma vez que, quanto mais se lê, mais aprimorada é a capacidade de uma leitura crítica (Carpenter, Miyake \& Just, 1995; Hannon \& Daneman, 2001).

Palabras-clave: Lectura. Técnica de Cloze. Desesperanza. Universidad.

A leitura representa o caminho de acesso ao pensamento crítico, visto que permite ao leitor a construção de novas informações e a formação de outras concepções. Ler criticamente requer uma conexão mental entre o sistema cognitivo e as partes que compõem o texto, pois a essência da leitura é a sua compreensão, uma vez que, quanto mais se lê, mais aprimorada é a capacidade de uma leitura crítica (Carpenter, Miyake \& Just, 1995; Hannon \& Daneman, 2001).
A habilidade de leitura funciona como uma retroalimentação sob a perspectiva da teoria do processamento da informação, isto é, o processamento cognitivo insere e armazena as informações lidas de modo que essa informação, uma vez processada, servirá de base para a compreensão de futuras informações ainda não conhecidas (Gilabert, Martinez, \& Vidal-Abarca, 2005; Sierra \& Carretero, 1996; Woolfolk, 2000). 
A compreensão da leitura não pode ser considerada um processamento simples. Essa habilidade exige a interação de uma série de redes e esquemas neurais que atuam dinamicamente de modo a promover a elaboração e a organização mental (Flippo, 1998).
A compreensão da leitura não pode ser considerada um processamento simples. Essa habilidade exige a interação de uma série de redes e esquemas neurais que atuam dinamicamente de modo a promover a elaboração e a organização mental (Flippo, 1998). A compreensão da leitura, por se tratar de um comportamento verbal complexo, tem sido foco de diversas investigações, permeadas pela teoria do processamento da informação.

Para Bzuneck (2004), esse processamento abrange o fato de a mente humana poder ser comparada, analogicamente, ao funcionamento de um computador. Nesse contexto, a aprendizagem se efetiva quando o estudante é capaz de abstrair e reutilizar alguma informação já armazenada de modo a aprimorar ou construir um novo conhecimento. O conhecimento prévio (background knowledge) armazenado pelo estudante, assume, portanto, grande importância (Dembo, 1988,1994).

Autores como Flippo e Caverly (1991) afirmam que os estudantes, sobretudo universitários, apresentam sérias limitações relacionadas à diversificação do conhecimento prévio. Para Oliveira e Santos (2005) e Sampaio e Santos (2002), no Brasil, muitos estudantes não conseguem compreender as informações básicas do texto ao ingressarem no ensino superior por apresentarem precário desenvolvimento da habilidade de leitura recebida anteriormente, o que revela leitores pouco críticos, sem habilidades argumentativas e com um baixo repertório para a resolução de problemas.

Maki, Schields, Wheeler e Zacchini (2005) destacam que a leitura e a sua compreensão são de essencial importância em todas as etapas do ensino. Oliveira e Santos
(2006) acrescentam que, no ensino superior, somente com um bom domínio dessa habilidade o estudante terá uma formação de qualidade e sairá com os conhecimentos técnicos necessários para uma boa atuação profissional.

Torna-se relevante conhecer mais acerca da compreensão da leitura dos estudantes universitários de forma a discutir as dificuldades, as facilidades, as motivações e os fatores que facilitam ou dificultam essa compreensão. O objetivo de se mensurar a compreensão da leitura é o de fomentar a possibilidade de oferecer ao estudante estratégias e materiais adequados, a fim de possibilitar um desempenho acadêmico positivo e auto-reforçador. Desse modo, com a preocupação de se mensurar a compreensão da leitura em universitários, foram realizadas pesquisas (Oliveira \& Santos, 2005, 2006, 2007; Oliveira, Santos \& Primi, 2003; Pinto, Alvarenga \& Kock, 1997; Santos, 1997; Santos, Primi, Taxa \& Vendramini, 2002; Suehiro, Santos \& Oliveira, 2004) que evidenciam o baixo repertório de conhecimento prévio e contextual, a baixa compreensão da leitura e mostram, também, que os estudantes que apresentam um bom desempenho acadêmico são aqueles que têm melhor compreensão da leitura.

Guterman (2003), Mason (2004) e Spira, Bracken e Fischel (2005) ponderam que a leitura e a sua compreensão também dependem de fatores internos diretamente associados a essa habilidade. O comportamento de ler envolve o uso de esquemas cognitivos, responsáveis pela atribuição de significado ao conteúdo lido. Desse modo, aspectos como motivação e atitudes positivas direcionadas à leitura facilitam a aquisição, o armazenamento e a posterior recuperação da informação lida. 
As atitudes e as emoções que permeiam a leitura formam uma situação complexa, e permitem o aparecimento de respostas de caráter afetivo-emocional. Portanto, as expectativas em relação aos benefícios da leitura ou até mesmo a algum resultado imediato e positivo, como obter um bom desempenho em uma avaliação, podem funcionar como modeladores de atitudes positivas direcionadas ao comportamento de ler (Mckenna, 1994).

Poucos estudos foram desenvolvidos com a finalidade de se levantar as atitudes de leitura de universitários ou de estudantes de outros níveis da educação formal. Dentre os estudos desenvolvidos, estão as investigações, apresentadas por ordem cronológica, de Parker e Paradis (1986), Castro e Souza (1996), Carelli, Bartalo, Cruz e Cordeiro (1999) e Oliveira, Santos e Oliveira (2007). De modo geral, esses estudos denunciam a carência de investigações sistemáticas e de projetos interventivos que visam a mensurar as atitudes em relação à leitura. Nem sempre o fato de o estudante valorizar a leitura significa que ele próprio apresente uma boa habilidade ou atitudes positivas direcionadas à leitura com um bom desempenho acadêmico.

Oliveira e Santos (2006) chamam a atenção para o fato de que muitos são os aspectos relacionados à aprendizagem do aluno, quais sejam, condições de estudo, conhecimentos da linguagem, experiências do indivíduo bem como a interação dos contextos físico, social e psicológico. Nesse sentido, levantase a hipótese de que, se os estudantes universitários apresentarem um bom controle cognitivo, com amplo repertório de experiências acadêmicas positivas, eles terão menor propensão a apresentar um estado emocional desequilibrado. Por outro lado, uma operação emocional malsucedida dificultará o processo de regulação, o que pode ocasionar algum transtorno emocional, tal como a desesperança, que demonstra que o indivíduo apresenta cognições negativas em relação à própria capacidade de realização. Autores como Neimeyer e Feixas (1992) associam a desesperança a um sintoma derivado dos estudos relacionados à depressão. Cunha (2001) afirma que a desesperança denota sensação de falta de sucesso e desprazer em relação ao futuro. As pessoas com esse quadro têm esquemas cognitivos disfuncionais, de modo que não esperam satisfação real ou não apresentam a esperança de um futuro melhor.

Beck e Steer (1993) sustentam que a desesperança representa uma sensação de forte pessimismo, que, muitas vezes, pode levar à depressão e ao suicídio (Chioqueta \& Stiler, 2007). Esse quadro é marcado por atitudes e cognições negativas que evoluem negativamente para a crença de que a melhor solução seria desistir, uma vez que o sentimento de autoderrota é predominante. Portanto, pode haver associação entre os sentimentos negativos, próprios da desesperança, e os traços de pessimismo e de baixas expectativas em relação ao futuro (pensamentos pessimistas). Assim sendo, o diagnóstico da desesperança pode ser um recurso valioso na prevenção do suicídio (O'Connor \& Cassidy, 2007).

Sob essa perspectiva, a desesperança está relacionada às percepções negativas e a um sentimento de fracasso e de derrota em relação a si próprio. Trata-se de um sentimento relacionado à sensação de fracasso no que se refere à realização de tarefas. Atividades que representam obstáculos e possibilidades de falta de êxito são abandonadas, o que produz impressões de impotência e de desesperança (Beck, Rush, Shaw, \& Emery, 1997). 
A universidade apresenta um ambiente competitivo para o estudante, pois este tem que apresentar um resultado satisfatório, com um bom desempenho na realização das tarefas. Muitos não conseguem responder de forma adequada às exigências impostas pelo ensino superior (Polydoro, Primi, Serpa, Zaroni \& Pombal, 2001; Vendramini et al., 2004). Portanto, aventa-se que, quando isso acontece, as expectativas são frustradas e, possivelmente, geram a sensação de fracasso, que se reflete negativamente na satisfação e no rendimento acadêmico ao longo do curso. Sendo assim, levanta-se a hipótese de que os estudantes que apresentam desesperança também são aqueles que apresentam atitudes negativas em relação à leitura e, por conseguinte, também demonstram uma compreensão de leitura pouco desejável. Pode-se, também, levantar a hipótese de que existam diferenças de desempenho em relação ao gênero, ao curso e à idade dos participantes. Nesta pesquisa, buscarse-á explorar essas diferenças, uma vez que pesquisas a esse respeito, considerando as variáveis aqui estudadas, ainda não foram desenvolvidas.

Nessa direção, os objetivos deste estudo foram levantar possíveis diferenças na compreensão da leitura, atitudes de leitura e desesperança em razão do gênero, curso e idade dos universitários. Também buscar-se-á levantar a possível relação entre compreensão de leitura, atitudes de leitura e desesperança.

\section{Método}

\section{Participantes}

Participaram 261 estudantes universitários dos cursos de radiologia $(11,5 \%, n=30)$, administração $(23,0 \%, n=60)$ e Psicologia $(65,5 \%, n=171)$, de universidades privadas dos Estados de São Paulo e Minas Gerais. A média de idade foi de 23 anos e 7 meses $(D p=6,8)$. A idade mínima foi de 17 anos, e a máxima, de 52 . O gênero masculino representou $31 \%(n=81)$ da amostra, e o feminino $68,6(n=179)$, e apenas uma pessoa $(0,4 \%, n=1)$ não informou o gênero.

Instrumentos

Para mensurar a compreensão da leitura, foi utilizado o Teste de Cloze (Taylor, 1953) em sua versão tradicional, em que se omitem todos os quintos vocábulos do texto, sendo que, no local da omissão, é colocado um traço de tamanho proporcional ao da palavra excluída. Para tanto, foi utilizado um texto adaptado de Luís Fernando Veríssimo (1995), intitulado Desentendimento, que continha 46 omissões e aproximadamente 250 vocábulos. Adotou-se o critério de correção literal, que considera como acerto o preenchimento da palavra exata que foi omitida. Assim, a pontuação no Cloze poderia variar de 0 a 46, considerando 0 para os erros e 1 para os acertos.

Utilizou-se a Escala de Atitudes de Leitura, baseada na versão elaborada por Silva e Naher (1981), que contém 30 afirmações, diante das quais os estudantes devem ler e assinalar com um ' $x$ ' o quanto concordam com cada uma. Apenas duas adaptações foram realizadas em relação à versão original, sendo a primeira referente à substituição da palavra escola por universidade, e a segunda, às alternativas de respostas; substituiu-se concordo fortemente por concordo plenamente (CP), e discordo fortemente por discordo plenamente (DP). As categorias concordo (C) e discordo (D) foram mantidas. Foram atribuídos 4 pontos para CP, 3 para C, 2 para D e 1 ponto para DP; o escore total é a soma da pontuação em cada item, sendo que a pontuação poderia chegar a 120 pontos. 
As Escalas Beck também foram utilizadas e traduzidas e adaptadas à realidade brasileira (Cunha, 2001), com permissão de The Psychological Corporation, U.S.A., por Jurema Alcides Cunha. Consistem de quatro escalas que mensuram sintomas de ansiedade, depressão, desesperança e ideação suicida (nesta pesquisa, somente foi empregada a Escala de Desesperança). Cabe destacar que o instrumento apresenta evidências de validade para a população adulta brasileira.

A Escala Beck de Desesperança apresenta 20 frases, nas quais o sujeito deve assinalar verdadeiro ou falso para cada afirmação apresentada. A classificação crítica de pessimismo, que indica a desesperança, varia de nível mínimo de desesperança (0-3), nível leve de desesperança (4-8), nível moderado de desesperança (9-14) e nível grave (superior a 14). Portanto, a pontuação da escala varia de 0 a 20.

Procedimento

Os cuidados éticos tomados na presente pesquisa estão em consonância com a Resolução no196/96, do Conselho Nacional de Saúde (2006). Assim sendo, um bom rapport foi estabelecido antes da aplicação dos instrumentos. A aplicação ocorreu de forma coletiva, em horário previamente agendado com cada instituição de ensino, para os alunos que haviam assinado o Termo de Consentimento Livre e Esclarecido. Cada aplicação durou aproximadamente 30 minutos em cada sala de aula. Vale esclarecer que, quanto à ordem de aplicação, aplicou-se, primeiro, o Teste de Cloze, seguido pela Escala de Atitudes de Leitura e pela Escala de Desesperança.

\section{Resultados}

Os dados foram organizados em planilha e submetidos à estatística descritiva e inferencial, conforme os objetivos deste estudo. Uma análise descritiva das pontuações obtidas nos instrumentos foi realizada. A Tabela 1 apresenta os dados.

Tabela 1. Distribuição das médias, desvios-padrão e pontuação máxima e mínima no Cloze, na Escala de Atitudes de Leitura e na Escala de Desesperança.

\begin{tabular}{lcccc}
\hline Medidas & $M$ & $D p$ & $\begin{array}{c}\text { Pontuação } \\
\text { mínima }\end{array}$ & $\begin{array}{c}\text { Pontuação } \\
\text { máxima }\end{array}$ \\
\hline Compreensão em leitura & 20,9 & 4,7 & 0 & 35 \\
Atitudes de leitura & 91,7 & 14,1 & 51 & 120 \\
Desesperança & 2,8 & 2,3 & 0 & 7 \\
\hline
\end{tabular}

Como se pode observar, a média de pontos da compreensão em leitura foi baixa, diferentemente da pontuação na escala de atitudes de leitura. Também foi possível levantar sintomas de desesperança. Na seqüência, visando a atender os objetivos deste estudo, serão apresentados os dados obtidos com a análise da diferença na pontuação no Cloze, na Escala de Atitudes de

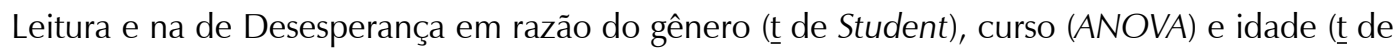
Student) dos estudantes. 
O teste $\underline{t}$ de Student foi utilizado para buscar diferenças na pontuação do Cloze, da Escala de Atitudes de Leitura e da de Desesperança em razão ao gênero dos participantes. Algumas diferenças estatisticamente significantes foram evidenciadas e podem ser observadas na Tabela 2.

Tabela 2. Valores de média, desvio-padrão, t e p obtidos com o teste t de Student no Cloze, na Escala de Atitudes de Leitura e na Escala de Desesperança, considerando os gêneros dos participantes.

\begin{tabular}{llcccc}
\hline Medidas & & $M$ & $D p$ & $\underline{\mathrm{t}}$ & $\underline{\mathrm{p}}$ \\
\hline Compreensão em leitura & Feminino & 21,4 & 4,7 & $-2,285$ & 0,010 \\
& Masculino & 19,8 & 4,6 & & \\
\multirow{2}{*}{ Atitudes de leitura } & Feminino & 93,3 & 14,4 & $-2,582$ & 0,008 \\
& Masculino & 88,4 & 13,2 & & \\
Desesperança & Feminino & 2,9 & 2,4 & 0,539 & 0,590 \\
& Masculino & 2,7 & 1,9 & & \\
\hline
\end{tabular}

Os dados da Tabela 2 revelaram que as meninas pontuaram mais nas diferentes medidas em relação aos meninos. O mesmo aconteceu na medida da desesperança, embora tal diferença não tenha sido significativa.

Recorreu-se à análise de variância - ANOVA para se aferir possíveis diferenças na pontuação dos instrumentos, considerando os cursos dos estudantes. Foi constatada diferença estatisticamente significativa entre os diferentes cursos no que se refere à compreensão da leitura e às atitudes em relação à leitura dos participantes, tendo em vista, respectivamente, $[F(2,258)=13,966$; $p=0,001]$ e $[F(2,528)=6,024 ; p=0,003]$. O teste post-hoc, de Tukey, indicou que a diferença na pontuação do Cloze estava entre os cursos de Psicologia $(M=21,6)$ e administração $(M=16,9)$ $(p=0,001)$ e administração e radiologia $(M=21,0)(p=0,001)$. Quanto à escala de atitudes de leitura, a diferença estava entre os cursos de Psicologia $(M=93,9)$ e radiologia $(M=87,2)(p=0,004)$. A média de pontos do curso de administração foi de 88,6 .

As diferenças na pontuação do Cloze, da Escala de Atitudes de Leitura e da Escala de Desesperança foram, então averiguadas, tendo em vista a idade dos participantes. Desse modo, as idades foram reunidas em 4 grupos. O primeiro foi composto por estudantes de 17 a 20 anos (36,0\%), o segundo grupo abarcou estudantes de 21 a 25 anos (38,7\%), no terceiro grupo estavam os estudantes de 26 a 35 anos (13,8\%) e o último grupo foi composto por estudantes com 36 anos ou mais $(7,7 \%)$. Apenas $3,8 \%$ das pessoas não informaram a idade. A ANOVA foi empregada mais uma vez, e, novamente, algumas diferenças estatisticamente significativas foram evidenciadas na compreensão da leitura, nas atitudes em relação à leitura e na desesperança dos participantes, considerando, respectivamente, $[F(3,248)=2,611 ; p=0,050],[F(3,248)=5,831 ; p=0,001] \mathrm{e}$ $[F(3,248)=3,130 ; p=0,026]$. A Tabela 3 apresenta os valores do teste post-hoc, de Tukey, no Cloze, na Escala de Atitudes de Leitura e na Escala de Desesperança, considerando a idade dos participantes. 
Tabela 3. Valores do teste post-hoc de Tukey no Cloze, na Escala de Atitudes de Leitura e na Escala de Desesperança, considerando a idade dos participantes.

\begin{tabular}{|c|c|c|c|}
\hline Medidas & $\begin{array}{c}\text { Grupos } \\
M\end{array}$ & $\begin{array}{c}\text { Grupos } \\
M\end{array}$ & $P$ \\
\hline Compreensão em leitura & $\begin{array}{c}2 \\
M=20,1\end{array}$ & $\begin{array}{c}4 \\
M=23,1\end{array}$ & 0,044 \\
\hline Atitudes de leitura & $\begin{array}{c}1 \\
M=88,2\end{array}$ & $\begin{array}{c}3 \\
M=95,5\end{array}$ & 0,041 \\
\hline & & $\begin{array}{c}4 \\
M=101,0\end{array}$ & 0,001 \\
\hline Desesperança & $\begin{array}{c}1 \\
M=3,2\end{array}$ & $\begin{array}{c}4 \\
M=1,5\end{array}$ & 0,014 \\
\hline
\end{tabular}

A ANOVA permitiu evidenciar que, tanto no Cloze quanto na Escala de Atitudes de Leitura, o grupo de estudantes mais maduros se saiu melhor em relação aos estudantes mais jovens. Contudo, no que tange à desesperança, o grupo de estudantes mais jovens demonstrou mais sintomas desesperançosos.

Buscou-se, também, uma possível correlação entre a pontuação no Cloze, na Escala de Atitudes de Leitura e na Escala de Desesperança. Assim sendo, a correlação de Pearson foi utilizada. Os dados revelaram correlação negativa e significativa entre a pontuação na Escala de Atitudes de Leitura e a pontuação na Escala de Desesperança $(r=-0,137$ e $p=0,027)$. No diz respeito aos escores do Cloze e à pontuação na Escala de Atitudes de Leitura, observou-se um p com valor baixo $(0,073)$, não podendo ser, contudo, considerado significativo. Já no caso da relação entre os escores do Cloze e a pontuação na Escala de Desesperança, o valor de $\underline{r}$ foi negativo e baixo $(-0,049)$, e o valor de p não foi significativo $(0,426)$, portanto, a correlação não foi estabelecida.

\section{Discussão e conclusão}

Os dados obtidos com a compreensão da leitura não foram positivos, uma vez que a média de acertos da população geral foi de 20,9 e nenhum participante conseguiu atingir a pontuação máxima de 46 pontos. Quando se considera a diferença na pontuação por gênero, a média do gênero feminino foi um pouco melhor $(M=21,4)$ do que a do gênero masculino $(M=19,8)$, contudo ambos os grupos estão muito aquém do nível de compreensão da leitura desejável para essa faixa de escolaridade. No caso das diferenças de pontuação por curso, verificou-se que os estudantes do curso de Psicologia $(M=21,6)$ se saíram melhor na compreensão da leitura e foram seguidos pelos estudantes do curso de radiologia $(M=21,0)$ e administração $(M=16,9)$, mas. ainda assim, não foi possível observar em nenhum dos grupos pelo menos uma média de acertos que se aproximasse da metade de acertos possíveis no teste de Cloze, que era de 23 pontos. Há que se mencionar as diferenças levantadas em razão da idade dos participantes, sendo que o grupo de estudantes com faixa etária dos 36 anos ou mais se saiu melhor na compreensão da leitura $(M=23,1)$, com diferença estatisticamente significativa em relação aos estudantes da faixa etária dos 21 anos aos $25(M=20,1)$. Nesse caso, os estudantes mais velhos conseguiram atingir pelo menos a metade de acertos possíveis no Teste de Cloze. 
Ainda a respeito dos dados obtidos, cabe esclarecer que não foram realizados estudos anteriores que tivessem investigado diferenças entre as três variáveis aqui analisadas, considerando o gênero, o curso e a idade dos estudantes universitários. Dessa forma, espera-se que a presente pesquisa possa contribuir para a realização de estudos futuros que visem a levantar e a tentar explicar tais diferenças. Apontamos essa limitação dos dados aqui trazidos e, na seqüência, discutiremos, à luz da teoria, a importância e os possíveis impactos da compreensão em leitura e das atitudes em relação à leitura no aprendizado do universitário.

Carpenter et al. (1995) e Hannon e Daneman (2001) são unânimes em considerar a importância de uma leitura com compreensão. Tendo em vista que os estudantes que participaram da presente pesquisa, em sua maioria, não conseguiram sequer atingir 23 pontos, que era a metade de acertos possíveis no Teste de Cloze, pode-se então supor que tais estudantes apresentam muitas limitações em sua compreensão textual, o que certamente irá depor desfavoravelmente em relação ao seu rendimento acadêmico (Flippo \& Caverly, 1991).

Algumas hipóteses que poderiam ser fomentadas em face desse desempenho na compreensão se referem ao fato de tais estudantes não apresentarem uma boa bagagem em termos de conhecimentos anteriores, o que resulta em uma leitura pouco crítica e também com pouca abstração de conteúdo (Sampaio \& Santos, 2002). Nesse sentido, pode-se supor que, se a leitura funciona com retroalimentação, conforme proposto por Gilabert et al. (2005), Sierra e Carretero (1996) e Woolfolk (2000), então há que se questionar se esses estudantes foram expostos, ao longo da vida, a um vasto repertório de informações que serviriam de base para uma boa compreensão.

Se a compreensão da leitura é algo tão importante no ensino superior e se funciona como um pré-requisito para uma formação profissional robusta (Oliveira \& Santos, 2006), então pode-se questionar como será a qualidade do profissional no mercado de trabalho, uma vez que, na universidade, este apresentava sérias dificuldades de compreensão da leitura e, portanto, dos conteúdos técnicos exigidos durante o curso. Desse modo, os dados obtidos neste estudo corroboram pesquisas anteriores sobre o tema (Oliveira \& Santos, 2005, 2006, 2007; Oliveira et al., 2003; Pinto et al., 1997; Santos, 1997; Santos et al. 2002; Suehiro et al., 2004) que evidenciaram a baixa compreensão textual dos estudantes universitários. Esse fato chama a atenção para a necessidade de adoção de medidas urgentes e mais diretivas das instituições de ensino, de modo que tais dificuldades sejam remediadas. Assim, há que se ressaltar a necessidade de cursos/ programas/disciplinas destinados a trabalhar a leitura na universidade e que estes sejam incorporados ao programas curriculares.

No que se refere às atitudes de leitura, a média de pontos foi de 91,7 , e o gênero feminino $(M=93,3)$ se destacou um pouco mais nas atitudes do que o masculino $(M=88,4)$. Quanto à idade dos participantes, os estudantes mais velhos $(M=101,0)$ apresentaram atitudes mais positivas em relação à leitura do que os estudantes das faixas etárias de 17 a 20 anos $(M=88,2)$ e de 26 a 35 anos $(M=95,5)$.

Esses resultados demonstram que os alunos participantes apresentaram atitudes positivas direcionadas à leitura e, provavelmente, essas atitudes podem estar associadas ao fato de que eles compreendem a importância da 
leitura para o seu aprendizado (Guterman, 2003; Mason, 2004; Spira et al., 2005). Além disso, os estudantes mais velhos, pela própria maturidade, demonstram mais atitudes positivas em relação à leitura justamente por atribuírem mais significado e importância à sua função em seu aprendizado.

Portanto, considera-se esse resultado positivo, uma vez que apresentar atitudes positivas em relação à leitura já aponta maiores possibilidades de que possíveis programas interventivos sejam bem aceitos pelos estudantes. Todavia, há que se ponderar que o fato de o estudante valorizar a leitura não significa necessariamente que ele goste ou leia bem. Assim, novamente, indica-se a necessidade de as universidades estimularem a leitura, não somente aquelas que respondem a um objetivo de conteúdo acadêmico mas também as que abordam aspectos sociais, políticos, históricos e diversão.

No que tange à desesperança, a média dos participantes foi de 2,8. Essa pontuação denota um nível de desesperança considerado mínimo. Os gêneros não se diferenciaram em relação ao sintoma de desesperança, mas houve diferença na média de pontos, tendo em vista a idade dos participantes. Os estudantes da faixa etária de 17 a 20 anos apresentaram maior incidência de sintomas desesperançosos $(\underline{M}=3,2)$ em relação aos estudantes de 36 anos ou mais $(\underline{M}=1,5)$. A classificação também pode ser considerada um nível mínimo de desesperança.

desesperança, mesmo que não alarmantes, podem ser vistos com cautela, uma vez que revelam

a incidência

de sentimentos negativos e pessimistas (Neimeyer \& Feixas, 1992). cognições e comportamentos associados à idéia de fracasso e à falta de perspectiva futura. Assim, aventa-se a hipótese de que, no caso do universitário que irá ingressar no mercado de trabalho e também ter uma profissão, esse quadro desesperançoso pode aumentar e atrapalhar o desempenho acadêmico desse estudante.

Um outro aspecto que merece atenção é a possibilidade de a desesperança levar à depressão e ao suicídio (Chioqueta \& Stiler, 2007). Portanto, cabe salientar que estudos que visem a mensurar a relação entre as variáveis ligadas à aprendizagem e à saúde mental deveriam ser desenvolvidos de modo que se possa conhecer melhor tais aspectos, possibilitando a ocorrência precoce do diagnóstico, o que irá melhorar a possibilidade de se obter êxito em uma intervenção. Mais uma vez, as universidades poderiam e deveriam dispensar mais atenção a esses aspectos.

A relação estabelecida entre as atitudes de leitura e a desesperança foi significativa e negativa. Sendo assim, quanto mais atitudes positivas direcionadas à leitura, menor é a pontuação na Escala de desesperança. Esse dado corrobora as considerações de O'Connor e Cassidy (2007), uma vez que esses autores também evidenciaram que, à medida que aumentam as expectativas/atitudes positivas, diminui a incidência de desesperança. No caso da compreensão da leitura e a pontuação na Escala de Desesperança, não houve relação significativa, muito embora o valor de $\underline{r}$ tenha sido negativo. Observa-se que a presente pesquisa somente explorou as variáveis aqui estudadas, mas novas pesquisas estão em andamento. Desse modo, concluímos que o estudo da saúde mental associado às habilidades acadêmicas na universidade ainda é um campo que necessita ser desbravado. 


\section{Considerações finais}

A universidade tem o dever de oferecer uma formação de qualidade ao seu corpo discente. Um modo de proporcionar essa formação seria investir em projetos psicoeducacionais a fim de remediar ou aprimorar habilidades cognitivas que não foram estimuladas, sobretudo quando se menciona os cursos universitários noturnos, em que o maior percentual de alunos é advindo de escolas públicas que deixaram muitas lacunas no seu aprendizado.

Nesse contexto, cabe o diagnóstico psicoeducacional de aspectos associados à cognição, à aprendizagem e à saúde mental do estudante universitário. Esse fato se torna ainda mais urgente quando se sabe que uma saúde mental desfavorável leva ao baixo rendimento acadêmico e também pode evoluir para quadros depressivos graves ou até mesmo para o suicídio.

Sob esse aspecto, há que se citar a necessidade da realização de investigações futuras que tenham por objetivo averiguar a ocorrência de quadros associados à depressão, à ansiedade, à desesperança e ao estresse, entre outras patologias que poderiam levar a alguma conseqüência negativa e drástica na vida acadêmica do universitário. Contudo, poucas são as pesquisas e as iniciativas práticas que se propõem a dar mais atenção à saúde mental do universitário.

Acrescente-se a isso o fato de haver pouco conhecimento sobre os aspectos afetivos e emocionais que permeiam a vida de nossos universitários de cursos de Psicologia. As dificuldades emocionais apresentadas por alguns estudantes no decorrer do curso não são trabalhadas ou tratadas pela universidade, pelo menos não na maior parte delas. Questionase se uma pessoa "emocionalmente doente" poderia oferecer atendimento psicológico seguro e eficaz.

De modo geral, as discussões sobre esse assunto ficam no campo subjetivo. Levanta-se a hipótese de ocorrência desse fato, visto que um atendimento mais voltado para a saúde mental do estudante universitário exigiria uma mobilização da instituição de ensino, com investimento em espaço adequado para o atendimento, em psicólogos treinados para fazer essa intervenção clínica, em material e em testes psicológicos, entre outros. Presumese que, embora as instituições tivessem que fazer um investimento significativo, este poderia se reverter de forma positiva na melhora do rendimento acadêmico dos estudantes. Seria ingênuo defender essa medida como solução para os problemas enfrentados pela educação superior no Brasil; contudo, talvez esse fosse um pequeno passo que poderia render resultados positivos.

Katya Luciane de Oliveira

Psicóloga, Mestre em Psicologia pelo Programa de Estudos de Pós-Graduação Stricto Sensu em Psicologia da Universidade São Francisco, Doutora em Psicologia, Desenvolvimento Humano e Educação pela Faculdade de Educação da UNICAMP, docente do Curso de Psicologia da Universidade São Francisco

Endereço para envio de correspondência:

Universidade São Francisco - R. Alexandre Rodrigues Barbosa, 45 - Centro Itatiba-SP - Brasil CEP: 13.251-900

E-mail: katya.oliveira@saofrancisco.edu.br katya_lincoln@ig.com.br

Recebido 19/10/2007 Reformulado 24/09/2008 Aprovado 25/09/2008 


\section{Referências}

Beck, A. T., \& Steer, R. A. (1993). Beck Hopeless Scale: Manual. San Antonio, TX: Psychological Corporation.

Beck, A. T., Rush, A. J., Shaw, B. F., \& Emery, G. (1997). Terapia cognitiva da depressão (S. Costa, trad.). Porto Alegre: Artes Médicas

Bzuneck, J. A. (2004). Aprendizagem por processamento da informação: uma visão construtivista. In E. Boruchovitch \& J. A. Bzuneck (Eds.), Aprendizagem: processos psicológicos e contextos social na escola (pp. 17-54). Petrópolis, RJ: Vozes.

Carelli, A. E., Bartalo, L., Cruz, V. A. G., \& Cordeiro, N. M. S. (1999). Leitura na universidade: resultados preliminares de um estudo. Manuscrito não publicado.

Carpenter, P. A., Miyake, A., \& Just, M. A. (1995). Language comprehension: Sentence and discourse processing. Annual Reviews Psychology, 46, 91-120.

Castro, R. V., \& Sousa, M. L. D. (1996). Hábitos e atitudes de leitura dos estudantes portugueses. In Resumos de Comunicações Cientificas, 16th World Congress on Reading. Praga.

Chioqueta, A. P., \& Stiler, T. C. (2007). Cognitives factors, engagement in sport and suicide risk. Archives of Suicide Research, 11(4), 375-390.

Conselho Nacional de Saúde. (2006). Resolução no 196. Diretrizes e Normas Regulamentadoras de Pesquisa. Recuperado em 19 de outubro de 2005, de http://www.conselho.saude.gov.br

Cunha, J. (2001). Manual em português das Escalas Beck. São Paulo: Casa do Psicólogo.

Dembo, M. H. (1988). Applying educational psychology in the classroom (3rd ed.). New York: Logman.

Dembo, M. H. (1994). Applying educational psychology (5th ed.). New York: Longman.

Flippo, R. F. (1998). Points of agreement: A display of professional unity in our field. The Reading Teacher, 52, 30-40.

Flippo, R. F., \& Caverly, D. C. (1991). College reading and study strategy programs. Newark, NJ: Ira.

Gilabert, R., Martínez, G., \& Vidal-Abarca, E. (2005). Some good texts are always better texts: Revision to foster inferences of readers with high and low prior background knowledge. Learning and Instruction, (15), 45-68.

Guterman, E. (2003). Integrating written metacognitve awarencess guidance as a psychological tool to improve student performance. Learning and Instruction, (13), 633-651.

Hannon, B., \& Daneman, M. (2001). A new tool for measuring and understanding individual differences in the component processes of reading comprehension. Journal of Educational Psychology, 93(1), 103-128.

Maki, R. H., Schields, M., Wheeler, A. E., \& Zacchini, T. L. (2005). Individual differences in absolute and relative metacomprehension accurancy. Journal of Educational Psychology, 97(4), 723-731.

Mason, L. H. (2004). Explicit self-regulated strategy development reciprocal questioning: Effects on expository reading comprehension among struggling readers. Journal of Educational_Psychology, 98(1), 209-218.

Mckenna, M. C. (1994). Towards a model of reading attitude acquisition. In E. H. Cramer \& M. Castle (Eds.), Fostering the love of reading: The affective domain in reading education. Newark, NJ: International Reading Association.

Neimeyer, R. A., \& Feixas, G. (1992). Cognitive assessment in depression: A comparison of existing measures. European Journal of Psychological Assessment, 8(1), 45-46.

O'Connor, R. C., \& Cassidy, C. (2007). Predicting hopelessness: The interaction between optimism/pessimism and specific future expectancies.Cognition \& Emotion, 21(3), 596-613.
Oliveira, K. L., Santos, A. A. A., \& Primi, R. (2003). Estudo das relações entre compreensão em leitura e desempenho em disciplinas na universidade. Revista Interação em Psicologia, 7(1), 19-25.

Oliveira, K. L., \& Santos, A. A. A. (2005). Compreensão em leitura e avaliação da aprendizagem em universitários. Psicologia: Reflexão e Crítica, 18(1), 118-124.

Oliveira, K. L., \& Santos, A. A. A. (2006). Compreensão de textos e desempenho acadêmico. Psic - Revista de Psicologia da Vetor Editora, 7(1), 19-27.

Oliveira, K. L., \& Santos, A. A. A. (2007). Estudo de intervenção para a compreensão em leitura e produção de textos na universidade. Manuscrito submetido à publicação.

Oliveira, K. L., Santos, A. A. A., \& Oliveira, E. Z. (2007). Estudo das propriedades psicométricas de uma escala de atitudes de leitura para universitários. Paradigma - Maracay, 28(2), 165-180.

Parker, A., \& Paradis, E. (1986). Attitude development toward reading in grades one through six. Journal of Educational Research, 79(5), 313-315

Pinto, C. J. A., Alvarenga, M. A. P., \& Kock, R. A. A. (1997). Hábitos de leitura e compreensão de texto entre universitários. In G. P. Witter (Ed.), Leitura e universidade (pp. 133-165). Campinas, SP: Alínea.

Polydoro, S. A. J., Primi, R., Serpa, M. N. F., Zaroni, M. M. H., \& Pombal, K. C. P. (2001). Desenvolvimento de uma escala de integração no ensino superior. Psico-USF, 6(1), 11-17.

Sampaio, I. S., \& Santos, A. A. A. (2002). Leitura e redação entre universitários: avaliação de um programa de intervenção. Psicologia em Estudo, 7(1), 31-38.

Santos, A. A. A. (1997). Psicopedagogia no 3o grau: avaliação de um programa de remediação em leitura e estudo. Pro-Posições, 8(1), 27-37.

Santos, A. A. A., Primi, R., Taxa, F., \& Vendramini, C. M. M. (2002). O Teste de Cloze na avaliação da compreensão em leitura. Psicologia: Reflexão e Crítica, 15(3), 549-560.

Sierra, B., \& Carretero, M. (1996). Aprendizagem, memória e processamento da informação: a Psicologia cognitiva da instrução. In C. Col, J. Palácios, \& A. Marchesi (Eds.), Desenvolvimento psicológico e educação: psicologia da educação (pp. 122-137, A. Mello Alves, trad.). Porto Alegre: Artes Médicas.

Silva, E. T., \& Naher, J. P. (1981). Questionário para avaliar atitudes de leitura de alunos de $1^{\circ}$ e $2^{\circ}$ graus. In Livro de Resumos - $3^{\circ}$ Congresso de Leitura do Brasil (pp. 19-26). Campinas, SP: UNICAMP.

Spira, E. G., Bracken, S. S., \& Fischel, J. E. (2005). Predicting improvement after first-grade reading difficulties: The effects of oral language, emergent literacy and behavior skills. Developmental Psychology, 41(1), 225-234

Suehiro, A. C. B., Santos, A. A. A., \& Oliveira, K. L. (2004). Habilidades em compreensão da leitura: um estudo com alunos de Psicologia. Estudos de Psicologia (Campinas), 21(2), $29-41$.

Vendramini, C. M. M., Santos, A. A. A., Polydoro, S. A. J., Sbardelini, E. T., Serpa, M. N. F., \& Natário, E. G. (2004). Construção e validação de uma escala sobre avaliação da vida acadêmica (EAVA). Estudos de Psicologia (Natal), 9(2), 259-268.

Taylor, W. L. (1953). Cloze procedure: A new tool for measuring read-ability. Journalism Quarterly, 30, 415-433.

Veríssimo, L. F. (1995). Desentendimento. Ícaro: Revista de Bordo da Varig, 11(136)

Woolfolk, A. (2000). Psicologia da educação. Porto Alegre: Artmed. 